\title{
Transmissibles et Fertilité
}

\section{Etude d'un modèle expérimental d'infection de l'appareil génital mâle}

Conséquences de l'injection de E. coli dans l'épididyme et la vésicule séminale du rat.

\author{
André CLAVERT et Claudine CRANZ
}

Groupe de recherche en Andrologie, Faculté de Médecine, Strasbourg

\section{RESUME}

L'injection d'E. coli uropathogène dans la queue de l'épididyme du rat entrấne dans $74,2 \%$ des cas une épididymite chronique qui s'accompagne d'une orchite nécrotique $(16,1 \%)$ ou de lésions testiculaires ou infectieuses $(45,2 \%)$. L'injection de bactéries tuées induit les mêmes lésions testiculaires non infectieuses, ces destructions sont vraisemblablement d'origine toxique.

L'injection de bactéries dans la vésicule séminale entraîne une vésiculite qui régresse dès le 7ème jour pour disparaître progressivement. Simultanément la prostate est envahie par des polynucléaires, cette infection persiste pendant plus de trois mois. L'injection d'E. coli associée à la castration bilatérale détermine la formation d'abcès vésiculaires chroniques.

L'étude des lymphatiques du tractus génital permet d'expliquer la diffusion des infections, de l'épididyme vers le testicule et de la vésicule séminale vers la prostate.

L'épididyme est un organe qui se défend mal contre l'infection alors que la vésicule séminale est capable, grâce à l'activité de son épithélium, de lutter contre l'infection, par contre la prostatite secondaire à la vésiculite est d'emblée chronique.
Mots clés : Infection expérimentale de l'appareil génitale mâle, épididymite, vésiculite, prostatite.

\section{INTRODUCTION}

En médecine de la reproduction, l'infection génitale chronique de l'homme pose de nombreux problèmes diagnostiques et thérapeutiques. En effet les infections aiguës, de diagnostic clinique relativement aisé, sont responsables d'obstructions épididymaires et déférentielles provoquant des azoospermies. Dans ce cas la relation entre infection et stérilité est aisée. Cependant dans de nombreux cas les infections chroniques, souvent cliniquement muettes, déterminent des hypofertilités ; dans ces cas la relation entre infection et stérilité est plus délicate. Ces hypofertilités plus ou moins sévères sont dues à :

- des lésions de la fonction épididymaire par destruction définitive de l'épithélium, les cellules épithéliales épididymaires ne se multipliant plus après la puberté [4],

- des modifications des sécrétions glandulaires avec modification du plasma séminal et perturbation de ses fonctions dans la maturation et la protection du spermatozoïde [2],

- l'apparition d'anticorps antispermatozoïde qui immobilise les spermatozoïdes [6],

- la présence de polynucléaires qui entraînent des peroxydations des acides gras de la membrane des spermatozoïdes,

- la présence de germes, ces germes pou- 
vant se fixer sur les spermatozoïdes, détruire leurs membranes et enrouler leurs flagelles [']

En outre les spermes d'hommes infectés ne sont pas utilisables en fécondation in vitro car ils infectent les milieux de culture.

Dans notre consultation de stérilité, nous avons trouvé sur 2000 cas, 20,2 \% d'anomalies du plasma séminal que l'on peut mettre en relation avec des lésions d'une des glandes du tractus; dans ces cas, $17,9 \%$ sont des réductions de la sécrétion prostatique. Dans $13 \%$ des cas nous avons trouvé des signes patents d'infection (polynucléaires, IgA, spermoculture positive).

Il nous a paru important de trouver un modèle animal pour comprendre comment une infection aiguë peut évoluer vers la chronicité. Nous avons choisi le rat pour sa facilité d'utilisation et $E$. coli car c'est le germe le plus fréquemment retrouvé dans les spermocultures; la souche choisie est k120 rencontrée dans les infections urinaires.

Nous présentons dans cet article la synthèse de plusieurs publications réalisées dans notre laboratoire.

\section{MATERIEL ET METHODES}

Chez des rat" Wistar" de $150 \mathrm{~g}$, anesthésiés au chloroforme, un volume de $0,2 \mathrm{ml}$ de milieu a été injecté, par voie chirurgicale, soit dans la lumière de la vésicule séminale, soit dans la queue de l'épididyme droit, le côté gauche servant de témoin ; dans certains cas une castration bilatérale a complété l'expérimentation.

Les animaux sont sacrifiés, $2,4,7,14,30$, 45,60 jours après l'injection.

Tout le tractus génital est prélevé, fixé, coupé et coloré pour une étude histologique.

Les lymphatiques sont mis en évidence par l'injection d'encre de chine, dans la queue de l'épididyme, la paroi de la vésicule séminale, le déférent et la prostate. Les animaux sont sacrifiés $2,6,24$ et 48 heures après l'injection.

\section{RESULTATS}

1. Injection de $10^{6}$ germes dans la queue de l'épididyme (LUCHETTA et Coll. 1983).

Sur 62 animaux traités, $16(25,8 \%)$ ne présentent aucune anomalie, $8(12,9 \%)$ présentent une épididymite isolée, $28(45,2 \%)$ une épididymite avec des lésions testiculaires non infectieuses (les tubes séminiferes présentent dès le second jour une exfoliation de l'épithélium séminifère, 14 jours après l'injection certains tubes sont totalement vides d'autres sont très remaniés, deux mois après le traitement les lésions persistent. Chez 10 animaux soit $(16,1 \%)$ nous avons observé une épididymite avec orchite nécrotique et infiltration leucocytaire péritubulaire massive. Nous n'avons jamais observé de diffusion de l'infection vers la tête de l'épididyme et le déférent, ce qui tend à montrer que la diffusion canalaire ne joue pas un rôle important dans la diffusion de l'infection. Il n'y a pas d'atteinte des voies génitales controlatérales.

\section{Injection de $10^{6}$ germes tués dans la queue de l'épididyme}

Dans 3 cas sur 7 cas, ce traitement a entraîné des lésions testiculaires non infectieuses, identiques à celles décrites dans l'expérience précédente.

3. Injection de $10^{6}$ germes dans la lumière de la vésicule séminale droite (MAGLION et Coll. 1986)

Pour cette expérimentation nous avons traité 20 rats.

Un jour après l'injection, la lumière de la glande est envahie par les polynucléaires, les cellules épithéliales présentent des vacuoles. Dans les jours qui suivent, de nombreuses cellules de cet épithélium glandulaire sont nécrotiques, mais à partir du $7^{\circ}$ jour l'épithélium se restaure. 
L'étude histologique montre que la concentration en polynucléaires ne diminue que secondairement à la restauration de l'épithélium. En outre une semaine après le traitement des traînées de polynucléaires apparaissent dans le tissu interstitiel prostatique, elles persistent pendant plus de deux mois alors que les polynucléaires ont disparu de la lumière de la vésicule séminale.

\section{Injection de $10^{6}$ germes tués dans la lumière de la vésicule séminale}

La lumière de la glande n'est pas envahie par les polynucléaires mais l'épithélium présente des destructions identiques à celles observées dans le cas de l'injection de germes vivants.

\section{Injection de germes dans la vésicule séminale avec castration bilatérale}

Dans ce protocole, sur 14 cas étudiés nous avons toujours obtenu la formation d'un abcès qui persiste jusqu'a 60 jours au moins. Cette évolution n'a jamais été observée chez les animaux non castrés.

\section{Etude des lymphatiques du tractus génital du rat (BAGNOLA et Coll. 1988)}

La figure 1 résume les résultats obtenus. L'épididyme est drainé par les mêmes voies que le testicule, des anastomoses existant entre l'épididyme et le testicule. Le déférent est drainé pour sa partie initiale vers l'épididyme et sa partie distale vers la prostate. La vésicule séminale est drainée vers la prostate.

L'encre de chine injectée dans la prostate reste en place pendant plus de 48 heures, le drainage est très faible, nous n'avons pas trouvé de ganglions contenant des particules d'encre de chine.

\section{DISCUSSION}

Les différentes glandes de l'appareil génital mâle ne se comportent pas de la même manière devant l'injection de la même quantité de $E$. coli. L'épididyme est incapable de se défendre; des abcès torpides se constituent, le tissu interstitiel devient scléreux et les épithéliums s'atrophient; dans certains segments l'épithélium est complètement détruit d'où le passage des germes dans le tissu sous muqueux, puis interstitiel.

La vésicule séminale par contre se défend bien. Les germes injectés détruisent l'épithélium et de nombreux polynucléaires envahissent la lumière de la glande, mais après 7 jours l'épithélium se régénère et le

Tableau 1 : Evolution des lésions microscopiques de la vésicule séminale et de la prostate après injection d'E. Coli dans la vésicule séminale.

\begin{tabular}{lllllllllll}
\hline JOUR & $\mathbf{1}$ & $\mathbf{2}$ & $\mathbf{3}$ & $\mathbf{4}$ & $\mathbf{7}$ & $\mathbf{1 0}$ & $\mathbf{1 5}$ & $\mathbf{3 0}$ & $\mathbf{4 5}$ & $\mathbf{6 0}$ \\
\hline VESICULITE & + & + & ++ & +++ & +++ & ++ & + & 0 & 0 & 0 \\
PROSTATITE & 0 & 0 & 0 & + & + & ++ & +++ & +++ & +++ & +++ \\
\hline
\end{tabular}

Tableau 2 : Effets de l'injection de E. Coli et E. Coli tués sur l'épithélium de la vésicule séminale.

\begin{tabular}{lcccccccccc}
\hline JOUR & $\mathbf{1}$ & $\mathbf{2}$ & $\mathbf{3}$ & $\mathbf{4}$ & $\mathbf{7}$ & $\mathbf{1 0}$ & $\mathbf{1 5}$ & $\mathbf{3 0}$ & $\mathbf{4 5}$ & $\mathbf{6 0}$ \\
\hline E. coli vivants & + & + & ++ & +++ & +++ & ++ & + & 0 & 0 & 0 \\
E. coli tués & + & + & ++ & ++ & 0 & 0 & 0 & 0 & 0 & 0 \\
\hline
\end{tabular}




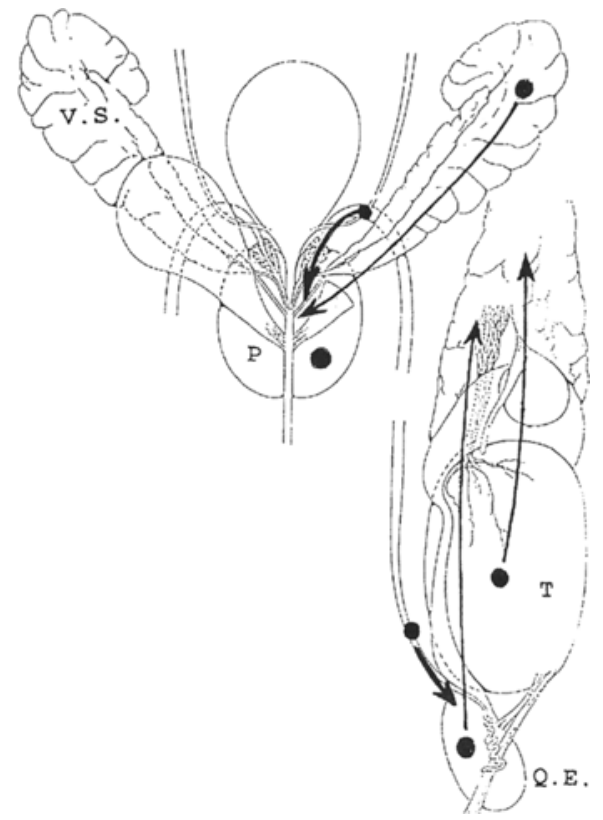

Figure 1 : Sont représentées sur cette figure les voies de diffusion de l'encre de chine à partir des points d'injection. Le testicule (T), la queue de l'épididyme (Q.E.) ainsi que la partie proximale du déférent sont drainés par les vaisseaux funiculaires. La vésicule séminale (VS) et la partie distale du déférent sont drainés par des vaisseaux lymphatiques vers la prostate ( $P)$. Qant à la prostate nous n'avons pas pu mettre en évidence de drainage, l'encre de chine reste pendant $48 \mathrm{H}$ dans la glande.

nombre de polynucléaires diminuent, 15 jours après la glande est libérée en grande partie de l'infection; il ne persiste que quelques polynucléaires dans le canal excréteur.

Il existe donc une différence très importante dans la résistance à l'infection, l'épididyme apparaît comme étant le plus fragile.

Pour la vésicule séminale, il apparaît clairement que la sécrétion est l'élément responsable de cette résistance aux germes; la régression de l'infection apparaît au moment de la régénération épithéliale ; en outre la castration, qui entraîne la disparition des sécrétions, détermine la formation d'abcès chronique.

La diffusion de l'infection dans le tractus génital ne se fait pas, dans notre cas, par voie canalaire. En effet dans l'épididyme, l'injection de germes dans la queue n'a jamais entraîné d'infection de la tête alors que des orchites nécrotiques sont observées. Des images de passage de l'infection par voie lymphatique ont pu être observées. L'étude des lymphatiques confirme ces observations, en effet il est possible de visualiser des lymphatiques qui passent du tissu interstitiel épididymaire dans l'albuginée.

Notre attention a été particulièrement attirée par les relations lymphatiques existant entre la vésicule séminale et la prostate. En effet la complication de la vésiculite aiguë est la prostatite chronique. Les germes passent directement du canal excréteur de la vésicule dans le tissu interstitiel prostatique. Les lymphatiques des vésicules séminales longent les canaux excréteurs puis le canal éjaculateur dans le tissu interstitiel prostatique. Il apparaît donc que la diffusion se fait par voie lymphatique.

L'injection d'encre de chine dans le tissu prostatique ne diffuse pas et cela pendant plus d'une semaine, cette observation est en faveur d'une grande pauvreté de la prostate en lymphatiques. Cette pauvreté en drainage lymphatique explique le passage des infections prostatiques à la chronicité.

Comme le montrent les expériences d'injection d'E. coli tués par la chaleur, l'effet toxique sur les épithéliums de l'épididyme et de la vésicule séminale explique en partie sa virulence et l'importance de la diffusion de l'infection. En outre les toxines de ce germe sont capables d'entraîner des destructions testiculaires qui apparaissent définitives. Ainsi ces infections peuvent être responsables de certaines hypofertilités inexpliquées rencontrées en clinique humaine. 
Les sécrétions du tractus génital constituent une barrière à la diffusion des germes vers l'épididyme et le testicule qui ne sont pas capables de se défendre. Lorsqu'une infection spécifique détruit les épithéliums des différentes glandes, la barrière antibactérienne tombe, permettant ainsi à des germes peu virulents d'atteindre l'épididyme et pénétrer dans le tissus sous muqueux entraînant le passage à la chronicité.

Ces observations expérimentales permettent d'émettre des hypothèses physiopathologiques quant à la fréquence élevée de prostatites chroniques dans la population des hommes hypofertiles. Ces expériences attirent également notre attention sur les effets toxiques des infections sur le testicule; il apparaît possible qu'un nombre important d'oligospermies puissent résulter de ce processus toxique ou d'un processus très voisin.

\section{CONCLUSION}

Le modèle animal que nous avons utilisé ne permet pas de tout expliquer, mais il est possible de tirer certaines conclusions :

- les glandes ne se défendent pas de la même manière contre l'infection, l'épididyme apparaissant comme le plus sensible.

- l'imprégnation hormonale joue un rôle important dans la mesure où elle contrôle la sécrétion des facteurs anti-infectieux participant à la constitution d'une barrière à la progression et la multiplication des germes.

- la diffusion de l'infection, dans notre modèle, se fait avant tout par voie lymphatique.

- les relations lymphatiques entre l'épididyme avec le testicule, et de la vésicule séminale avec la prostate, explique la diffusion privilégiées des infections entre ces organes.

- la prostate est mal drainée par les lymphatiques ce qui explique le passage fréquent à la chronicité.

\section{REFERENCES}

1. Bagnola J., Nieva H., Clavert A., Cranz C., BolLACK C. : Papel de los limfaticos en la difusion de la infection del tracto genital masculino. Ach. Esp. Urol. 1988, 41, 265-267.

2. Clayert A., Cranz C., Tardieu J.C. : Conséquences des infections du tractus génital sur la fertilité. In" Les voies génitales" ed. Dadoune, Clavert, ArvisDouin, Paris, 1994, 131-136.

3. Gavelida M., Lipovac V. : Effect of leucocytes on hypoosmotic swelling test of human sperm. Arch. Androl., 1993, 30, 55-61.

4. Lucchetta G., Clavert A., Meyer J.M., Bollack C. : Acute experimental E. coli epididymitis in the rat and its consequences on spermatogenesis. Urol. Res. 1983, 14, 265-266.

5. Maglion M., Nardi A., Cranz C., Clavert A., BolLACK C. : Acute vesiculitis and its prostatique complications caused by $\mathrm{E}$. coli in the rat. Urol. Res. 1986, 14, 265-266.

6. Pradignad A., Demand R.M., Cranz C., Clavert A. Antispermatozoal antibodies and genital infection. Urol. Int. 1991, 46, 18-21.

7. Robillard I., Meyer C., Ladame D., Cranz C., ClaVERT A. : Plasma séminal, bacteries et spermatozö̈des. Contracept. Fertil. Sex. 1993, 21, 380-381.

\section{ABSTRACT}

An experimental model for Infection of the male genital tract
A. Clavert, C. Cranz

Injection of uropathogenic E. coli into the rat cauda epididymidis results in chronic epididymitis in $\mathbf{7 4 . 2} \%$ of cases, accompanied by necrotic orchitis (16.1\% of cases) or non-infectious testicular lesions (45.2\% of cases). Injection of killed bacteria caused the same noninfectious testicular lesions, most probably due to toxic damage. Injection of bacteria into the seminal vesicles caused a vesiculitis which regressed after seven days and disappeared progressively. Simultaneously, the prostate was invaded by polymorphonuclear leucocytes, an infection which persisted for more than three months. E. coli injection in association with bilateral cas- 
tration gave rise to the formation of chronic vesicular abscesses. Examination of the male genital tract lymphatic system allowed explanation of the spread of infection from the epididymis to the testis, and from the seminal vesicle to the prostate. The epididymis is an organ with poor defense against against infection whereas the seminal vesicle is able, owing to the activity of its epithelium, to fight infection. On the other hand, prostatitis secondary to vesiculitis is immediately chronic.

Keys words : Infection, experimental, male tract, epididymis, vesiculitis, prostatitis. 